\title{
Fortschritte bei Multipler Sklerose
}

n dem Verständnis der Multiplen Sklerose hat sich in den letzten Jahren ein tief greifender Wandel vollzogen. Auch wenn ihre Ätiologie heute nicht vollständig geklärt ist, ist es doch gelungen, einige wesentliche Fortschritte vor allem auf den Gebieten der Histopathologie, der klinischen Neuroimmunologie und der Bildgebung zu erzielen, die in Ihrer Konsequenz sowohl zu einer Revision der klinischen Diagnosekriterien als auch zu einer Fortentwicklung der therapeutischen Möglichkeiten geführt haben.

So kann aufgrund übereinstimmender histologischer und kernspintomografischer Daten aus postmortalen und klinischen Untersuchungen davon ausgegangen werden, dass von Anfang an die für die Demyelinisierung verantwortliche entzündliche Komponente auch eng mit einer axonalen Schädigung verknüpft ist. Die Schädigung der Nervenstrukturen beginnt schon weit vor der ersten klinischen Manifestation und bei der Läsionsentstehung werden bereits die Weichen für oder gegen eine Regeneration gestellt. Die neuen Erkenntnisse haben sich in den Behandlungsempfehlungen der Multiple-Sklerose-Therapie-Konsensus-Gruppe (MSTKG) für den deutschsprachigen Raum niedergeschlagen. Die Grundlage für eine rechtzeitige Therapie ist die frühzeitige und exakte Diagnosestellung im klinischen Alltag, deren Kriterien auf einer internationalen Konsensuskonferenz festgelegt wurden. Diese nach Ian McDonald, dem Vorsitzenden der Expertenkommission, benannten Kriterien beziehen insbesondere die Kernspintomografie zur Diagnosesicherung mit ein. Der genau definierte Nachweis einer Krankheitsaktivität im zentralen Nervensystem kann bei Patienten mit erstmaliger klinischer Manifestation die Bestätigung durch einen zweiten Schub ersetzen.

Durch die Einführung der neuen immunmodulativen, den natürlichen Krankheitsverlauf der MS beeinflussenden Therapien, haben sich die Behandlungsmöglichkeiten der MS entscheidend verbessert. Die heute in Deutschland zugelassenen unterschiedlichen Präparate ermöglichen eine individuelle Abstimmung der Therapie und eine Eskalation im Sinne des von der MSTKG vorgeschlagenen Stufenschemas.

Mit der Entwicklung neuerer Therapieansätze und der Option einer rechtzeitigen Behandlung wird auch die Frage nach der Lebensqualität der Patienten neu gestellt. Zugleich rücken hiermit verbunden auch die bislang eher stiefmütterlich behandelten, nicht unmittelbar mit physischen Einschränkungen verbundenen Begleitsymptome dieser Erkrankung in den Vordergrund der Betrachtung. Tatsächlich ist schon seit langem bekannt, dass es im Rahmen einer MS zu Beeinträchtigungen der geistigen Leistungsfähigkeit sowie zu Persönlichkeitsveränderungen kommen kann. Erst in neuerer Zeit jedoch wurde im Rahmen systematischer Studien deutlich, dass kognitive Störungen nahezu die Hälfte der MS-Patienten betreffen. Gemeinsam mit Müdigkeit und Fatigue habe diese Symptome einen deutlichen Einfluß auf den Alltag und das Berufsleben der Betroffenen. Eine erhebliche psychosoziale Belastung stellen auch die im Rahmen dieser Erkrankung beobachtbaren affektivemotionalen Dysfunktionen dar. Diese werden im Einzelfall von den Patienten und/oder Angehörigen im weiteren Krankheitsverlauf als Persönlichkeitsveränderungen erlebt. Es wird zunehmend deutlich, dass diese Defizite sowohl vor dem Hintergrund psychosozialer Rahmenbedingungen als auch in Abhängigkeit prämorbider Persönlichkeitsdispositionen und der damit verbundenen Möglichkeiten der Krankheitsverarbeitung sowie als direkte Folge einer zentralnervösen Schädigung zu interpretieren sind. Dementsprechend und diesen Entwicklungen folgend erscheint es gerechtfertigt, den Fokus innerhalb dieser Schwerpunktausgabe auch auf die psychosozialen Aspekte dieser Erkrankung zu setzen.

Wir hoffen, mit den Beiträgen dieser Schwerpunktausgabe sowohl auf aktuelle, relevante Entwicklungen der MS hinzuweisen, als auch die Diskussion insbesondere im alltagsrelevanten psychosozialen Bereich zu beleben.
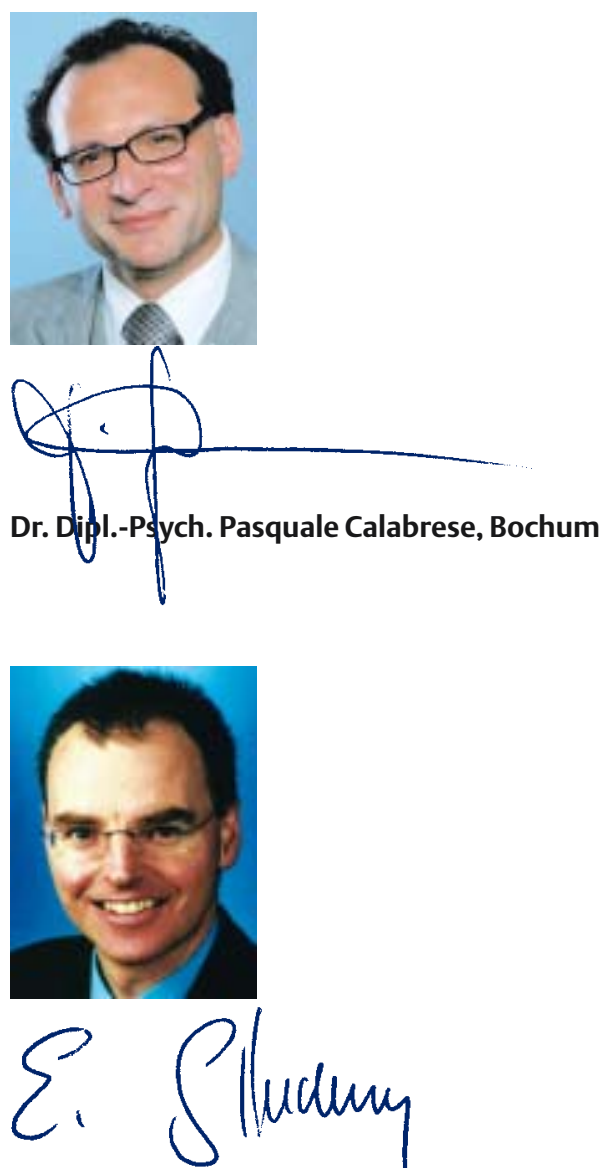

PD Dr. Eckhart Sindern, Bochum 